\title{
$\begin{array}{ll}\text { Research Square } & \text { Preprints are preliminary reports that have not undergone peer review. }\end{array}$ or referenced by the media as validated information. \\ Terahertz Waveguide-Based Pulse-Shaper System Analysis and Modeling
}

seyed mohammadreza razavizadeh ( $\nabla$ razavizadeh@ee.kntu.ac.ir)

K N Toosi University of Technology Faculty of Electrical Engineering https://orcid.org/0000-0002-62629512

\section{Ramezanali Sadeghzadeh}

K N Toosi University of Technology Faculty of Electrical Engineering

\section{Zahra Ghattan kashani}

K N Toosi University of Technology Faculty of Electrical Engineering

\section{Research Article}

Keywords: terahertz, pulse shaping, pulse compression, transfer function, waveguide

Posted Date: August 5th, 2021

DOI: https://doi.org/10.21203/rs.3.rs-688227/v1

License: (c) (1) This work is licensed under a Creative Commons Attribution 4.0 International License. Read Full License

Version of Record: A version of this preprint was published at Optical and Quantum Electronics on April 6th, 2022. See the published version at https://doi.org/10.1007/s11082-022-03558-2. 


\title{
Terahertz Waveguide-Based Pulse-Shaper System Analysis and Modeling
}

\author{
Seyed Mohammadreza Razavizadeh • \\ Ramezanali Sadeghzadeh • Zahra \\ Ghattan Kashani
}

Received: date / Accepted: date

\begin{abstract}
In this paper, the transfer function of a passive waveguide-based terahertz pulse shaper is achieved using the time domain data provided by the full-wave simulation of the structure. The fractional order of the transfer function is determined based on the time response resulting from an arbitrary excitation of the proposed pulse shaper. The full-wave electromagnetic numerical analyses are applied to attain the time-domain output data of the helical gold-ribbon dielectric-lined waveguide as the terahertz pulse shaper. In order to verify the simulation results, the proposed device has been examined using two different numerical methods which are the Finite Element Method (FEM) and the Finite Integral Technique (FIT). A good agreement was found between the results of FIT and FEM methods. The use of the system transfer function to analyze the structure is preferable to the full-wave simulation because of saving the execution time. Once the transfer function is determined, one could apply it for the subsequent time-domain analysis of the pulse shaper with various inputs.
\end{abstract}

Keywords terahertz $\cdot$ pulse shaping $\cdot$ pulse compression $\cdot$ transfer function · waveguide

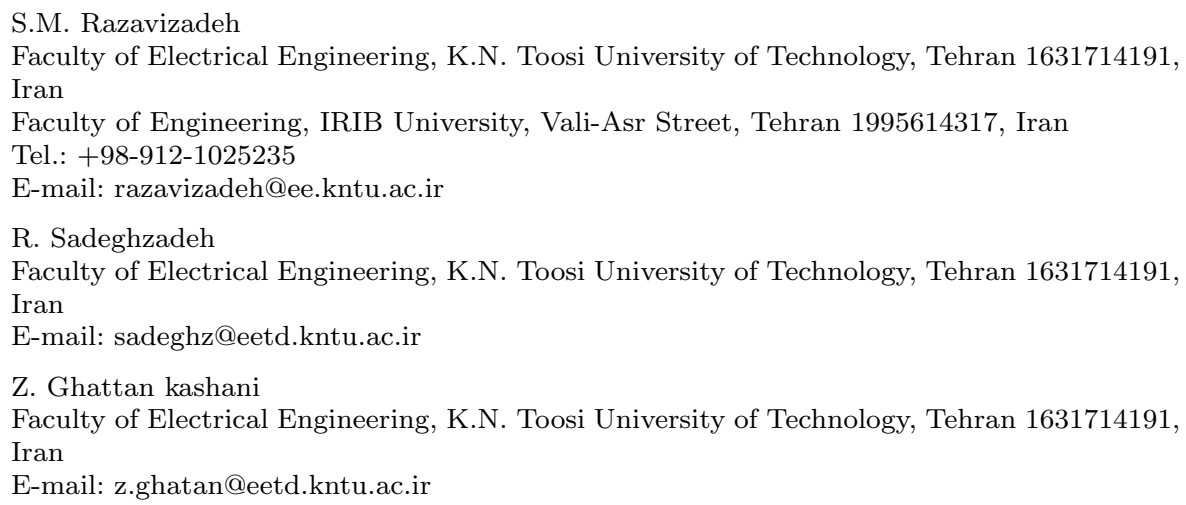




\section{Introduction}

Advances in the field of terahertz $(\mathrm{THz})$ science and technology have made impressive contributions to a variety of research areas including physics, material science [1]-[4], chemistry [5], and electrical engineering [6]-[9] for several decades. Recently, Veli and et al. [10] present diffractive layers as a modular pulse shaping network, which is used to shape an arbitrary broadband input terahertz pulse into square pulses with temporal-width using deep learning technique. After deep learning-based training of the machine, the thickness profiles of the resulting diffractive layers have been determined. Their proposed diffractive networks have been synthesized with various square terahertz pulses with duration from 10.58 ps to 15.69 ps. One essential drawback of such structures is bulky size of the pulse shaper (four layers with $3 \mathrm{~cm}$ space distance between sequential layers) which is not applicable to incorporation with compact semiconductor terahertz sources. Recently, in our previous work [11], we demonstrated a tunable $\mathrm{THz}$ pulse shaping technique utilizing the positive group velocity dispersion (GVD) region of a dielectric-lined circular waveguide loaded with a helical graphene ribbon. The results presented in [11] indicated a feasibility of tunable pulse compression applications by using the graphene ribbon. Here, we made a step forward for a passive fixed pulse-shaper by replacing the helical graphene ribbon with a gold ribbon. Compared with our previous work [11], the pulse shape is greatly improved by reducing the pedestal and ringing. Moreover, in order to prove the validity of the numerical analysis, the proposed device has been examined using Finite Element Method (FEM) and the Finite Integral Technique (FIT). The achieved results of FIT and FEM methods are in close agreement. The need for system identification in engineering is interesting [12], because of its ability to describe phenomena, which are not able to be fully explained by analytic approaches in complex problems. Fitting a transfer function (TF) or complex impulse response (CIR) is often used in modern wireless communications in order to characterize wireless channels [13]-citeR16. The advantage of such a response is to improve the performance of the channel with monitoring the transient responses of the system to various input signals over time-efficient signal processing operations. Examples include the analyses of electromagnetic waves in the presence of complex geometrical boundaries between normal and dispersive materials inducing a transient effect on propagating waves. System identification, which represents the transfer function, allows a great deal of flexibility in building mathematical models by using measurements of the system's input and output signals in terahertz spectrum [17]-[19]. Although transfer functions are generally used in the analysis of systems such as single-input single-output filters in the fields of signal processing, control theory, and communication theory, it could also be applied to provide comprehensive system models of electromagnetic problems. 


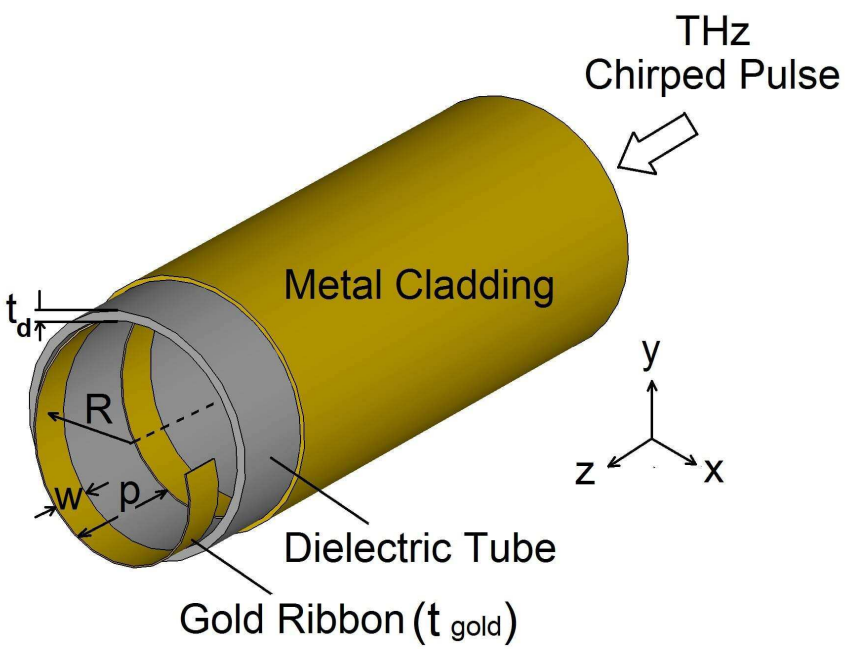

Fig. $13 \mathrm{D}$ geometry and side view of an internally dielectric-coated hollow-core waveguide loaded by a helical gold ribbon.

\section{Model Geometry and Materials}

Figure 1 sketches the passive waveguide-based terahertz pulse shaper. A gold ribbon of thickness $t_{\text {gold }}(1 \mu \mathrm{m})$, width $\mathrm{w}(41 \mu \mathrm{m})$, and pitch size $\mathrm{p}(126 \mu \mathrm{m})$ is helically wrapped and inserted inside a metalized dielectric tube with inner radius $\mathrm{R}$ of $100 \mu \mathrm{m}$ and thickness $t_{d}$ of $8.5 \mu \mathrm{m}$. By carefully choosing the geometric parameters and the dielectric material, an optimum condition for an arbitrary dispersion profile, large mode confinement with minimum mismatch, and propagation loss can be achieved. High-density polyethylene (HDPE) is used for the dielectric tube given its good THz transparency $(n=1.524$, and $\alpha=0.2 \mathrm{~cm}^{-1}$ at $\left.1 \mathrm{THz}[20]\right)$. The dielectric tube is completely covered by a gold film layer as a metal cladding. Gold is modeled with the Drude parameters as follows: $\epsilon_{\infty}=9.1, \omega_{p}=1.38 \times 10^{16} \mathrm{rad} / \mathrm{s}$, and $\Gamma=1.075 \times 10^{14}$ $\mathrm{Hz}[21]$.

\section{Numerical Modeling Methods for Time-Domain Data Acquisition}

Numerical techniques could be useful in theoretical studies of more challenging electromagnetic problems such as complex structures where exact analytical solutions are difficult to obtain. In this paper, our proposed device has been analyzed using FEM and FIT in order to prove the accuracy of the numerical analysis. Figure 2a,b show the transmittance and absorption coefficients of the proposed device across the whole operating bandwidth $(2.14-2.34 \mathrm{THz})$ for the transverse electric dominant mode $\left(T E_{11}\right)$. The phase difference between the input and the output signals of the 2-port device is expressed by the phase of the transmission coefficient $\left(S_{21}\right)$ as depicted in Figure 2c. The phase of 
the transmission coefficient, which is numerically calculated using both aforementioned numerical methods, demonstrates the dispersion characteristics of the signal transmission. In fact, it determines how the input signal is delayed when it travels between the input and output ports of the device in the desired frequency spectrum. A passive pulse compressor can be designed in a portion of electromagnetic spectra where the normalized group velocity $\left(V_{g r} / c\right)$ profile has a large negative gradient [22]-cite, R23, where $c$ denotes the speed of light in free space. $V_{g r}$ is inversely proportional to group delay (GD) when the propagation length (L) is constant as given in Equation (1). On the other hand, GD is defined versus the phase of the transmission coefficient $(\theta)$ as stated in Equation (2) [24]-[25].

$$
\begin{gathered}
\frac{V_{g r}}{c}=\frac{1}{300} \frac{L(\mu m)}{G D(p s)} \\
G D(p s)=-\frac{1}{360} \frac{\partial \theta(\mathrm{deg})}{\partial f(T H z)}
\end{gathered}
$$

As shown in Figure 3a, the proposed structure shows the normalized group velocity profile with a relatively large negative gradient in the frequency range of $f_{L o}$ to $f_{H i}$ which is equal to $2.14-2.34 \mathrm{THz}$. Furthermore, we provide a comprehensive time-domain analysis that verifies the overall pulse-compression performance of the proposed device. Figure $3 \mathrm{~b}$ depicts the output envelope waveforms of the proposed device based on a chirped-input signal obtained using FIT and FEM The chirped-input signal is a y-polarized chirped sine function, as stated in Equation (3):

$$
E_{y}=\left\{E_{0} \sin \left(2 \pi f_{H i} t-\mu t^{2}\right), \quad \text { if } t \leq T_{0} 0,\right. \text { andotherwise }
$$

where $f_{H i}$ is the upper-band frequency of the negative slope of the GV profile $(2.34 \mathrm{THz}), T_{0}$ is the input pulse width, $E_{0}$ is the electric field amplitude, and $\mu$ is the chirp factor. The chirp factor for a uniform input pulse spectrum in the negative slope of the GV region from $f_{L o}$ to $f_{H i}, 2.14-2.34 \mathrm{THz}$, can be calculated using Equation (4):

$$
\mu=\frac{\pi\left(f_{H i}-f_{L o}\right)}{T_{0}}
$$

As the simulation results exhibit, there is good agreement between the results of FIT and FEM methods over the entire operating bandwidth (2.14$2.34 \mathrm{THz}$ ). Based on theory, it is evident that the energy of electromagnetic waves in a dispersive medium travels in a group velocity profile, which is dependent on the frequency just as our proposed structure is, which is shown in Figure 3a. Therefore, if such a dispersive structure is excited by using a linearly down-chirped pulse like $\mathrm{E}_{y}$, which is introduced in Equation (3), it can be expected in a group velocity profile with negative gradient in terms of the frequency spectrum the front pulses with lower group velocity travel with 


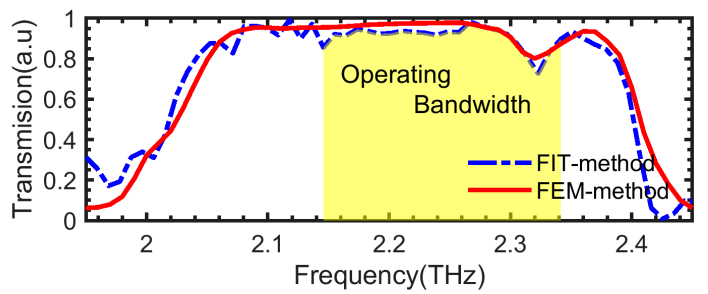

(a)

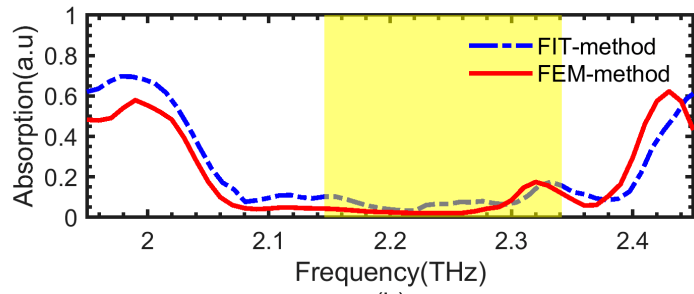

(b)

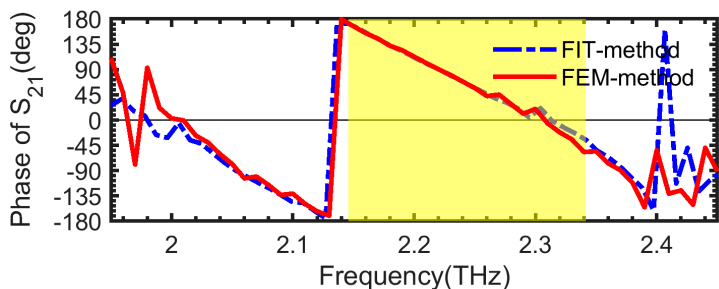

(c)

Fig. 2 (a) Transmittance, (b) absorption spectra, and (c) the phase of $S_{21}$ of the proposed device using Finite Integral Technique (FIT) and Finite Element Method (FEM).

larger delay of $\mathrm{T}_{d, H i}$ than the tail of the input pulses with delay of $\mathrm{T}_{d, L o}$ as described in Equations (5) and (6) [26].

$$
\begin{aligned}
& T_{d, H i}=\frac{L_{0}}{\left.V\right|_{f_{H i}}} \\
& T_{d, L o}=\frac{L_{0}}{\left.V\right|_{f_{L o}}}
\end{aligned}
$$

If the input chirped-pulse duration is chosen as $\left(\mathrm{T}_{0}=\mathrm{T}_{d, H i}-\mathrm{T}_{d, L o}\right)$, one can expect that the tail of the input pulse travel will overtake the front one. Based on the numerically obtained information of group velocity, it is shown in Figure 3a that we obtained the optimal traveling length of $\mathrm{L}_{0}$ as $579 \mu \mathrm{m}$. Figure 3a compares the normalized group velocity and the group delay of the proposed structure with those reported in [11]. It is clearly seen that the graphene-based pulse shaper in an equal bandwidth around its center frequency, as shown in Figure $3 \mathrm{a}$, is lower $\Delta \mathrm{V}_{g}$ than the Au-based one. 


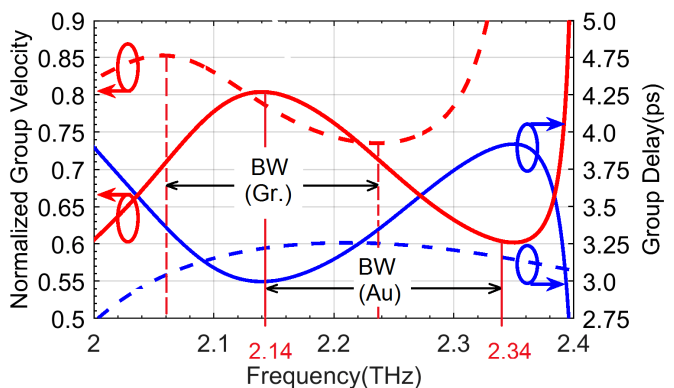

(a)

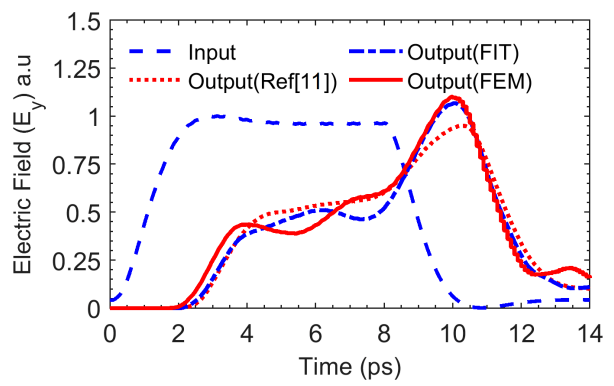

(b)

Fig. 3 (a) Group delay and normalized group velocity extracted from the phase of the transmission coefficient using FIT method for the proposed structure (solid line) and the zero-biased graphene-based structure reported in [11] (dashed line), and (b) the output envelope waveforms of the proposed device based on a chirped-input signal using two different numerical approaches of FIT and FEM, compared with output signal of a zero-biased graphene-based one [11].

This effect leads to the longer optimal length $(720 \mu \mathrm{m})$, and consequently, more transmission loss in the graphene-based pulse shaper. This comparison reveals how the material dispersion contributes to the total chromatic dispersion of the proposed structure. On the other hand, the simplicity is very important in microscale optical device fabrication. The main challenge in our previous work reported in [11] was the design complexity due to the use of graphene and the bias facilities including $\mathrm{SiO}_{2}$ spacer and gold-bias electrodes. Therefore, a compact and simple structure able to be incorporated with fibers, signal processors, terahertz time-domain spectroscopy (THz-TDS) systems, and etc. is introduced in this work to relax the structure complexity. Moreover, Figure $3 \mathrm{~b}$ shows that the compressed pulse at the optimal position of the output probe $\left(\mathrm{L}_{0}\right)$ of the proposed structure has a narrower-shaped pulse with the lower pedestal compared with that of the zero-biased graphene-based structure introduced in [11]. As shown in Figure 4, the maximum compressed pulse shape is obtained at the output of a y-polarized electric field, which is located at the distance of $575 \mu \mathrm{m}$ from the input reference plane of the proposed device. Figure $4 \mathrm{a}$ shows the traveled input pulses just after traveling $50 \mu \mathrm{m}$, about $10 \%$ of 
the optimal length while Figure $4 \mathrm{~b}$,d illustrate the probe outputs located at $525 \mu \mathrm{m}$ and $625 \mu \mathrm{m}$, before and after the optimal position $\left(L_{0}=575 \mu \mathrm{m}\right)$. These figures clearly show how the probe output signal starts to grow to a peak at the tail position before the optimal position, while after passing the optimum length, the temporarily narrowed pulse peak starts to downgrade. The $3 \mathrm{D}$ demonstration of the spatial-temporal electric field distribution along the proposed waveguide device can be used to characterize the field confinement performance for future applications such as material detection sensors or time-domain spectroscopy (TDS). Figure 5 gives a comprehensive description of the y-polarized electric field distributions at the optimal probe position in three important moments of the compressed pulse duration, assigned as $\mathrm{t}_{A}$, $\mathrm{t}_{B}$, and $\mathrm{t}_{C}$, which correspond to peak, pedestal, and ringing positions (see Figure 5a). As depicted in Figure 5b-d, the field distribution of the peak moment $\left(\mathrm{t}_{A}\right)$ is significantly more confined than pedestal $\left(\mathrm{t}_{B}\right)$ and ringing $\left(\mathrm{t}_{C}\right)$ moments. This ensures the proposed device can be employed as an efficient pulse shaper for various pulse-based $\mathrm{THz}$ applications including fast data communications, TDS, and imaging. Another important conclusion from the electric field distribution would be the mode purity of the proposed structure at the fundamental propagation linear polarized mode pattern of circular waveguide $\left(\mathrm{TE}_{11}\right)$.

In the remainder of this section, we briefly show the effects of each parameter of the proposed structure on the power transmission and the dispersion performance. Figure 6a shows how the thickness of the Au-ribbon, as helically varying dispersive boundary conditions, influences the GV profile of the proposed structure as well as the insertion loss. The dispersion characteristics of the structure have more dependence with $t_{\text {gold }}$ in the lower part of the operating frequency band than elsewhere. Moreover, the return loss of the structure can be tuned effectively within the target operating bandwidth in order to reach the lowest value with the highest uniformity. Figure $6 \mathrm{~b}$ shows the internal dielectric coating thickness $t_{d}$ has no significant effect on the GV, especially in the region with negative gradient, while the insertion loss has been kept lower than $-0.4 \mathrm{~dB}$. The dispersion and transmission performance of the proposed structure have significant sensitivity to the most important parameters of the helix which are the gold ribbon width, $w$, and the pitch size $p$, which can be seen in Figure $6 \mathrm{c}-\mathrm{d}$. Figure $6 \mathrm{c}$ illustrates the GV profile is more sensitive to the gold ribbon width below the optimum value, while with a slightly wider ribbon, the GV profile remains unchanged around the center frequency. In addition, for a larger or shorter pitch size, the negative gradient of GV becomes steeper with a down-frequency shift.

In order to ensure that the transient (ringing and pedestal) section has no significant effect on the overall time domain output pulse response, the proposed structure has been simulated in three different fixed lengths of 800 $\mu \mathrm{m}, 1000 \mu \mathrm{m}$, and $1400 \mu \mathrm{m}$, as shown in Figure $7 \mathrm{a}-\mathrm{c}$. The resultant output signals at the optimal position from the input port, $\mathrm{L}_{0}$, have been achieved by applying a unique input signal defined with Equation (3). The outcomes show stable responses without any significant deformation which could be used for 


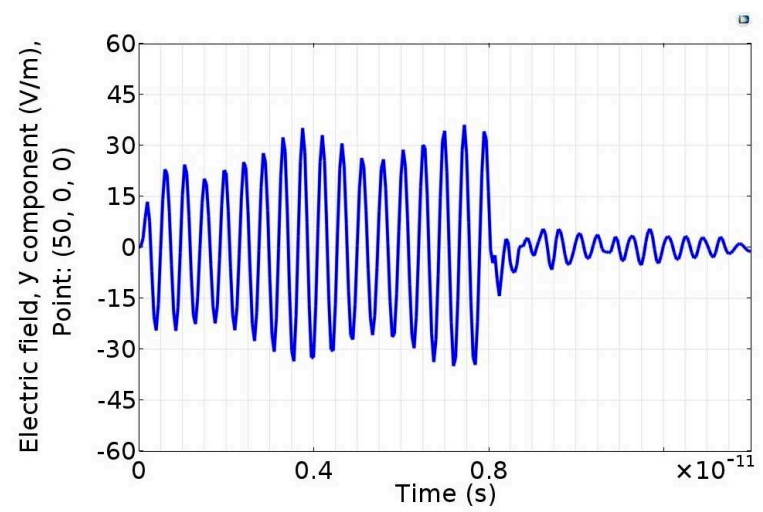

(a)

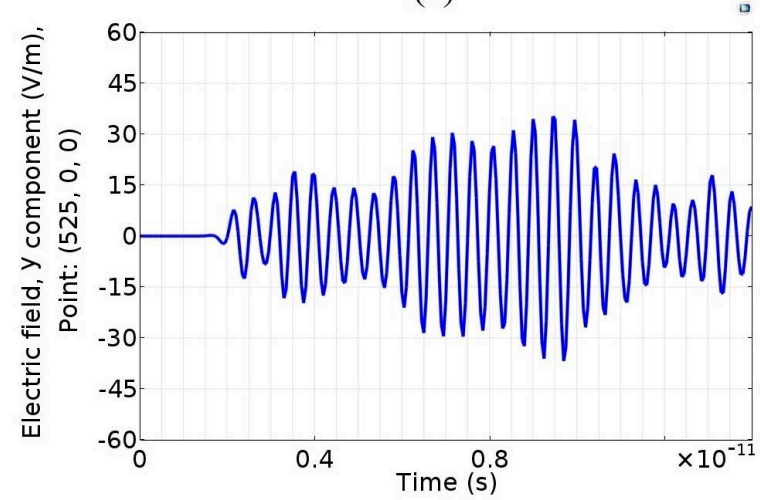

(b)

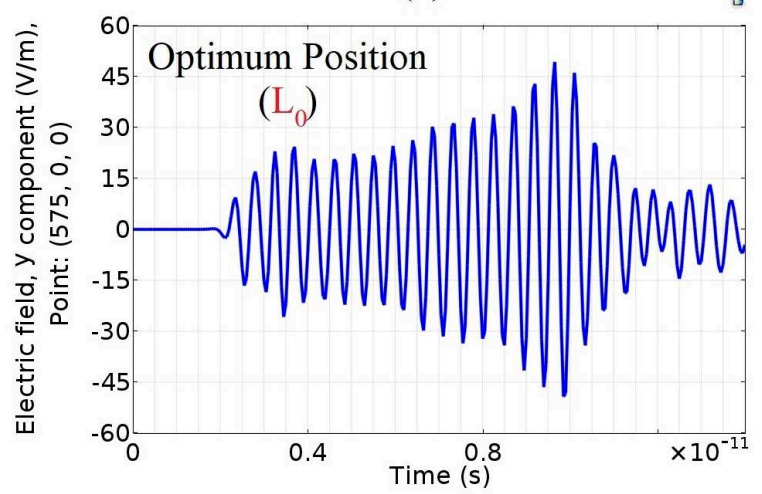

(c)

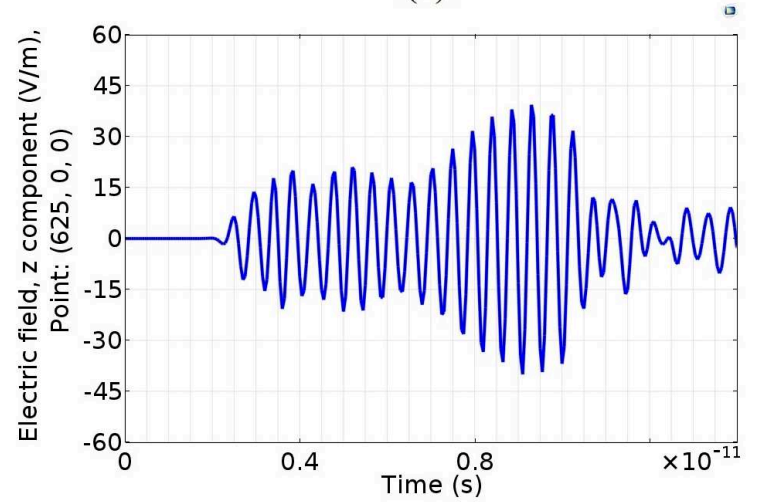

(d)

Fig. 4 The output y-polarized E-field probe signals at various positions of the proposed structure $(\mathbf{a})$ front position $(\mathrm{z}=50 \mu \mathrm{m}),(\mathbf{b})$ before, and $(\mathbf{d})$ after optimal length $(575 \mu \mathrm{m})$, and finally, at (c) $\mathrm{L}_{0}$. 


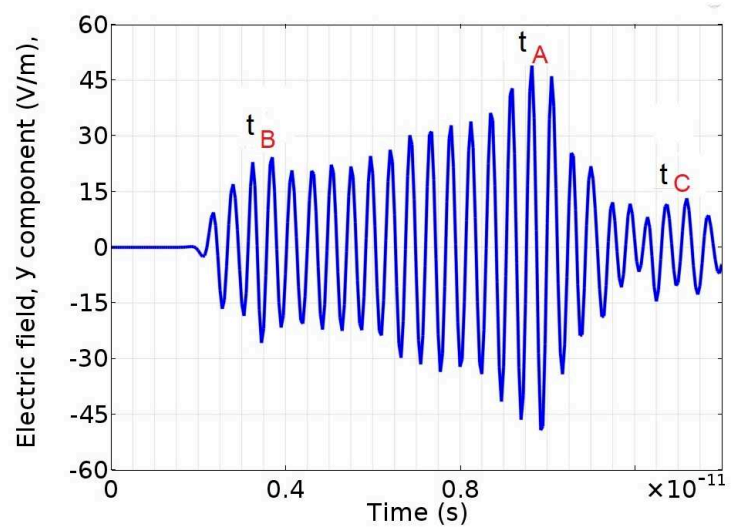

(a)
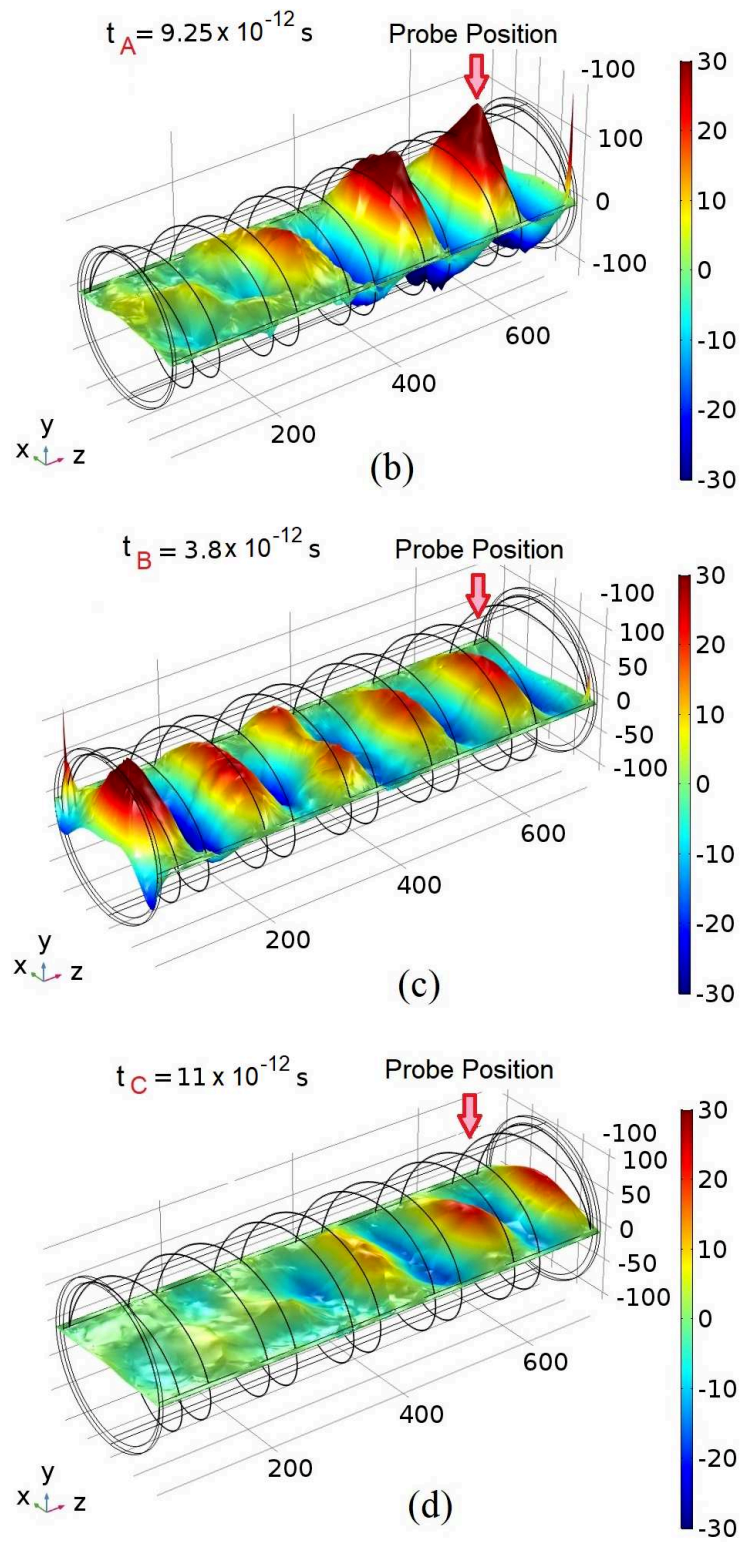

Fig. 5 The electric field distributions at the optimum positions and the corresponding peak time positions of (b) $\mathrm{t}_{A},(\mathbf{c}) \mathrm{t}_{B}$, and $(\mathbf{d}) \mathrm{t}_{C}$ as marked on (a) the probe signal output. 


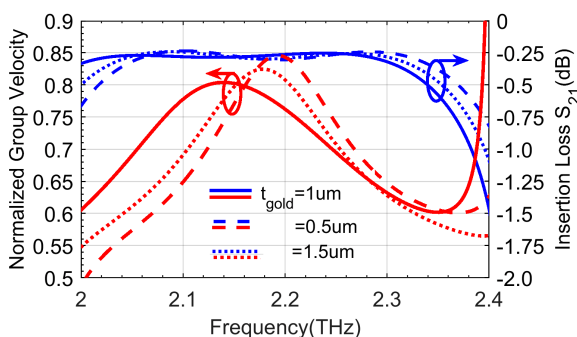

(a)

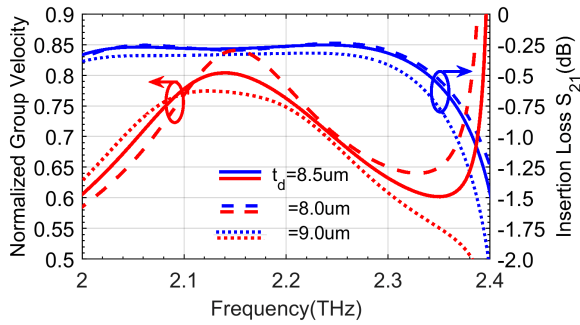

(b)

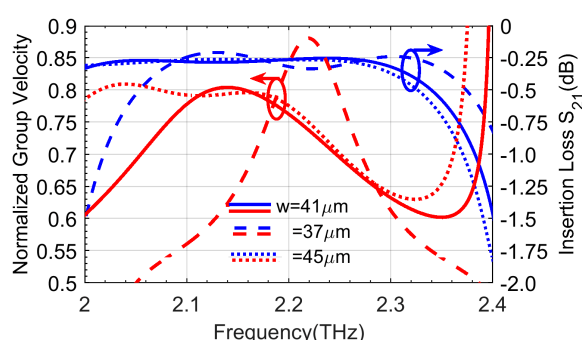

(c)

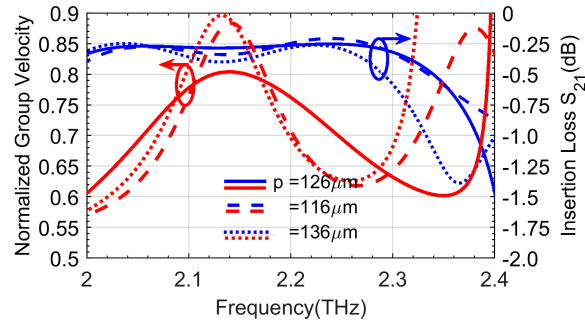

Fig. 6 Effect of (a) gold-ribbon thickness, (b) dielectric thicknesses, (c) helix width and (d) pitch size of the helix on the insertion loss and the normalized group velocity $\left(\mathrm{V}_{g} / \mathrm{c}\right)$.

example for high speed board-to-board interconnecting $\mathrm{THz}$ systems [27]. We can also show that when the structure is excited by an input signal consisting of two sequential chirped pulses, the transient section has no significant effect on the final output compared with that of the single input pulse. This achievement confirms the applicability of the structure to high speed data communications (see Figure 8).

The next section will describe how to estimate the transfer function of the proposed structure from the time-domain simulation response.

\section{Transfer Function Estimation}

A transfer function is an important notion in signal processing, which allows one to relate an output signal and an input. This section focuses on the estimation of the general transfer function of the proposed device. In pulse shaping, knowledge of the transfer function makes it possible to achieve the temporal output pulse from any arbitrary input pulses. By incorporating the full wave time-domain simulations (obtained in the previous section) and the numerical transfer function estimation approach, it becomes possible to extend the application of the system theory to a wider range of optical problems. The proposed waveguide-based structure is assumed as a linear time invariant (LTI) system which is stable and can be described by a transfer function of the form 

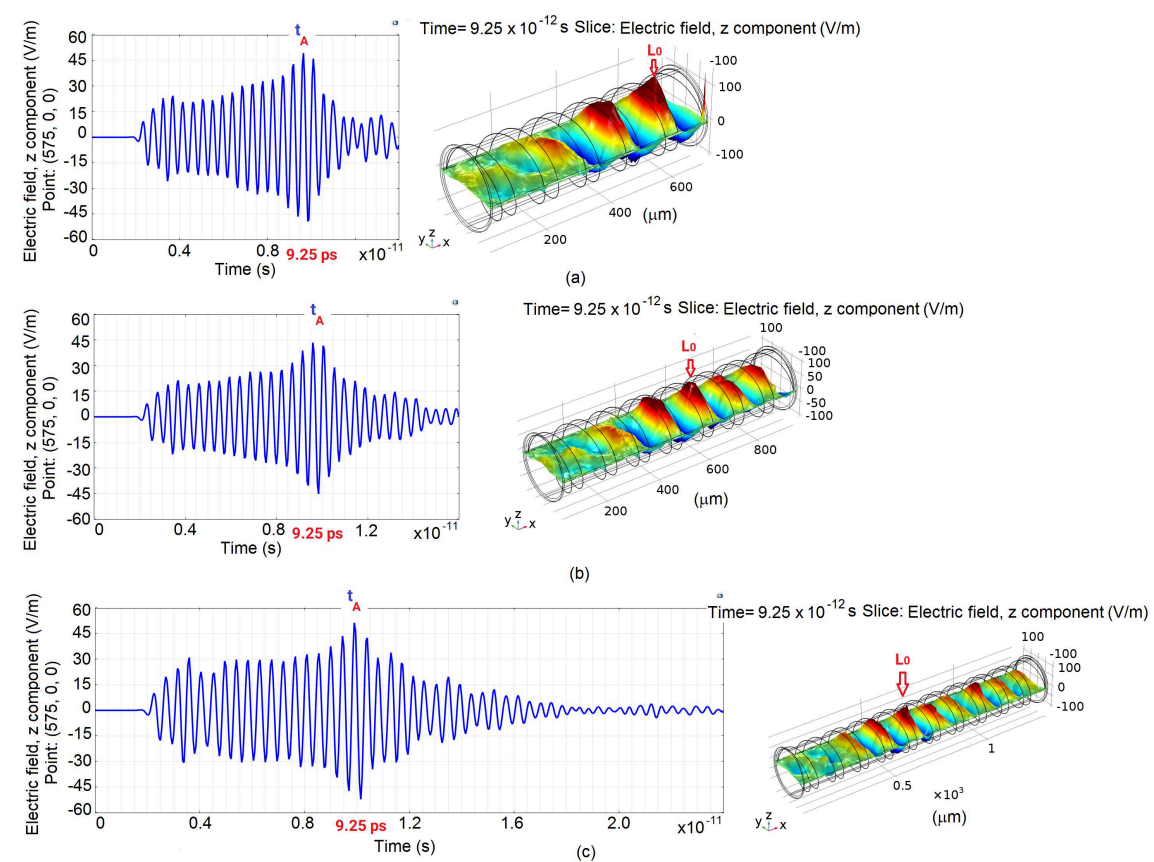

Fig. 7 The output signal at the optimal position $575 \mu \mathrm{m}$ for three different lengths of (a) $800 \mu \mathrm{m},(\mathbf{b}) 1000 \mu \mathrm{m}$ and (c) $1400 \mu \mathrm{m}$ of the proposed structure.

Equation (7):

$$
H(s)=\frac{b_{0}+b_{1} s^{\beta_{1}}+\ldots+b_{m} s^{\beta_{m}}}{a_{0}+a_{1} s^{\alpha_{1}}+\ldots+a_{n} s^{\alpha_{n}}}
$$

where $b_{0}, b_{1}, \ldots, b_{m}, a_{0}, a_{1}, \ldots, a_{n}$ are the coefficients to be estimated, and $\beta_{0}$, $\beta_{1}, \ldots, \beta_{m}, \alpha_{1}, \alpha_{0}, \ldots, \alpha_{n}$ are the positive real valued exponents. The proposed identification approach is based on the transfer function estimation using the input/output temporal data which are generated in the full-wave transient simulation of the proposed waveguide under a chirped-input signal. In this work, the MATLAB signal processing toolbox was employed to estimate the transfer function coefficients given in Table 1 based on the obtained time samples of the compressed signal. Figure 9 presents the input-chirped signal and the results of the estimated transfer function and those obtained by the FEM simulation. It is noteworthy that the coefficients of the numerators and denominators of $\mathrm{H}(\mathrm{s})$ with four zeros and nine poles are determined so that the highest similarity between the FEM output, Figure $9 \mathrm{~b}$, and the reconstructed one by using H(s), Figure 9c, has been achieved.

The accuracy of applying the estimated transfer function for calculating the output waveform can be verified based on the responses of the proposed structure to two different input signals. The first one is a nonchirped sinusoidal pulse while $\mu$ is set to zero and $f_{H i}$ is replaced with the center operation frequency of $f_{0}\left(\left(f_{H i}+f_{L o}\right) / 2\right)$ in Equation (3) and the second one is the well- 

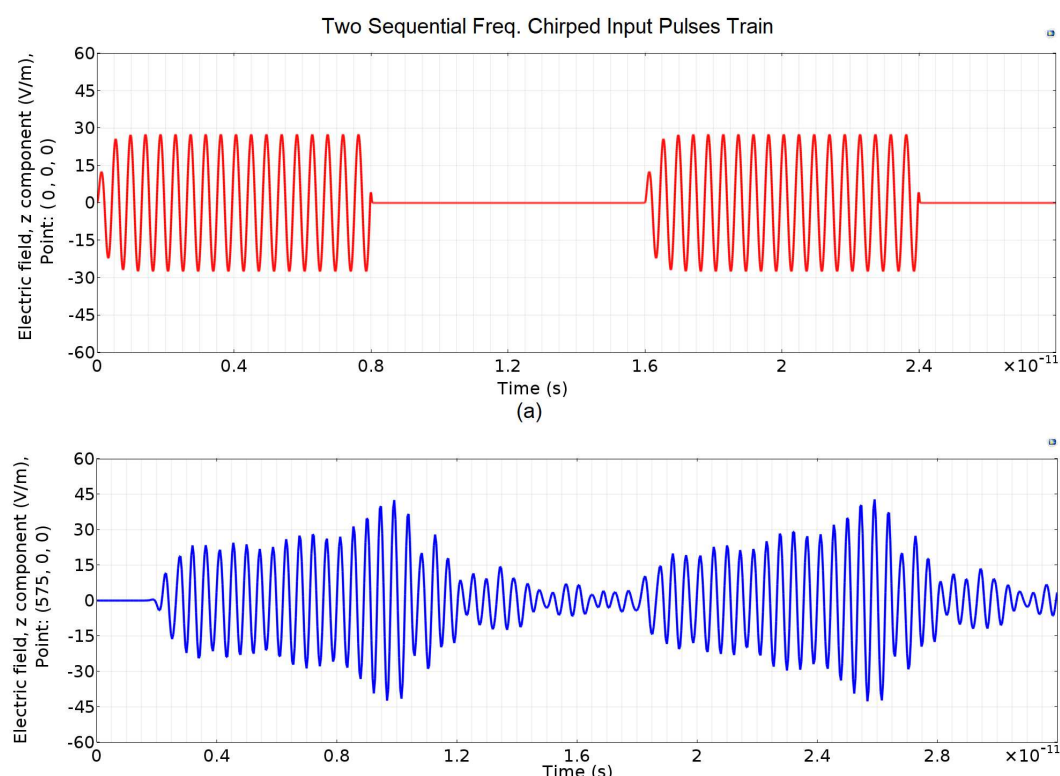

(b)

Fig. 8 (a) The excitation input signal consisting of two sequential chirped pulses, and (b) corresponding output signal at the optimal position of $575 \mu \mathrm{m}$, computed using FEM

known broadband Gaussian pulse which is defined as Equation (9). Figure 9a,b show a time-domain validation test of $\mathrm{H}(\mathrm{s})$ introduced by the coefficients given in Table 1. A good agreement between the full-wave results achieved by the FIT simulation and those of applying the equivalent system function, $\mathrm{H}(\mathrm{s})$, is observed.

$$
E_{y}= \begin{cases}E_{0} e^{-\left(\left(t-0.5 T_{0}\right) / \tau\right)^{2}} \sin \left(2 \pi f_{0} t\right), & \text { if } t \leq T_{0} \\ 0, & \text { otherwise }\end{cases}
$$

where $\tau$ is $2 \mathrm{ps}$ for an appropriate bandwidth compatible with the proposed structure and $T_{0}$ is set to $8 \mathrm{ps}$, the same as previously used in Equation (3). As shown in Figures 10a,b, this approach has great accuracy and could replace the full-wave simulation analysis which is very time consuming. In fact, the TF estimation with the features of the wideband and memory-efficient analysis could be applied in conjunction with other complicated model estimation techniques such as Transmission-Line (TL) models and narrow band equivalent circuit models.

The accuracy of applying the estimated transfer function for calculating the output waveform can be verified based on the responses of the proposed structure to two different input signals. The first one is a non-chirped sinusoidal pulse while $\mu$ is set to zero and $f_{H i}$ is replaced with the center operation frequency of $f_{0}\left(\left(f_{H i}+f_{L o}\right) / 2\right)$ in Equation (3) and the second one is the well- 


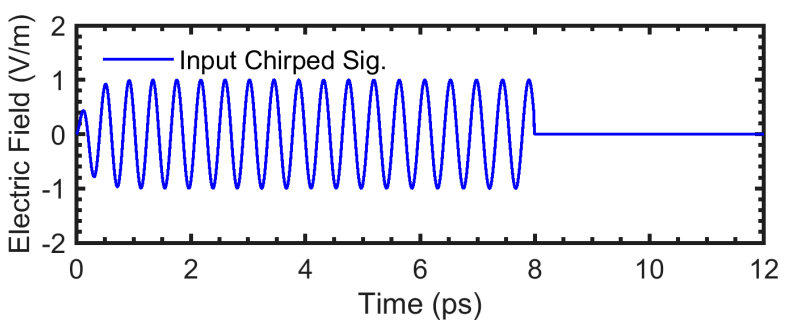

(a)

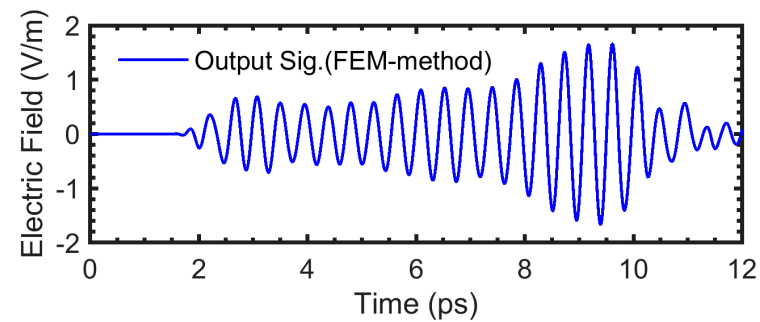

(b)

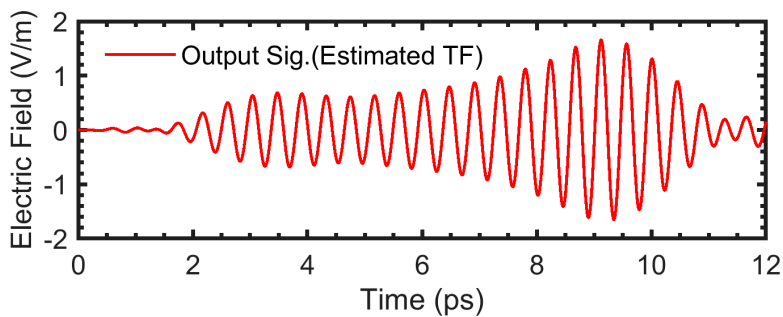

(c)

Fig. 9 (a) Chirped-input signal, (b) reference output signal obtained from the FEM analysis, and (c) output signal obtained from estimated transfer function model of the proposed device.

Table 1 The coefficients of the numerators and denominators of the estimated transfer function $\left(\beta_{m}=\mathrm{m}\right.$, and $\alpha_{n}=\mathrm{n}$, where $\mathrm{m}$ and $\mathrm{n}$ are integer numbers $\left.1,2, \ldots, 9\right)$.

\begin{tabular}{ccccc}
\hline$b_{0}$ & $b_{1}$ & $b_{2}$ & $b_{3}$ & $b_{4}$ \\
\hline$-7.17 \times 10^{113}$ & $2.8 \times 10^{100}$ & $-2.7 \times 10^{88}$ & $4.9 \times 10^{74}$ & $-2.5 \times 10^{62}$ \\
\hline$b_{5}$ & $b_{6}$ & $b_{7}$ & $b_{8}$ & $b_{9}$ \\
\hline $1.4 \times 10^{115}$ & $6.7 \times 10^{102}$ & $1.3 \mathrm{e} \times 10^{90}$ & $5.3 \times 10^{77}$ & $4.7 \times 10^{64}$ \\
\hline$a_{0}$ & $a_{1}$ & $a_{2}$ & $a_{3}$ & $a_{4}$ \\
\hline $1.4 \times 10^{115}$ & $6.7 \times 10^{102}$ & $1.3 \times 10^{90}$ & $5.3 \times 10^{77}$ & $4.7 \times 10^{64}$ \\
\hline$a_{5}$ & $a_{6}$ & $a_{7}$ & $a_{8}$ & $a_{9}$ \\
\hline $1.5 \times 10^{52}$ & $7.0 \times 10^{38}$ & $2.0 \times 10^{26}$ & $3.8 \times 10^{12}$ & 1 \\
\hline
\end{tabular}



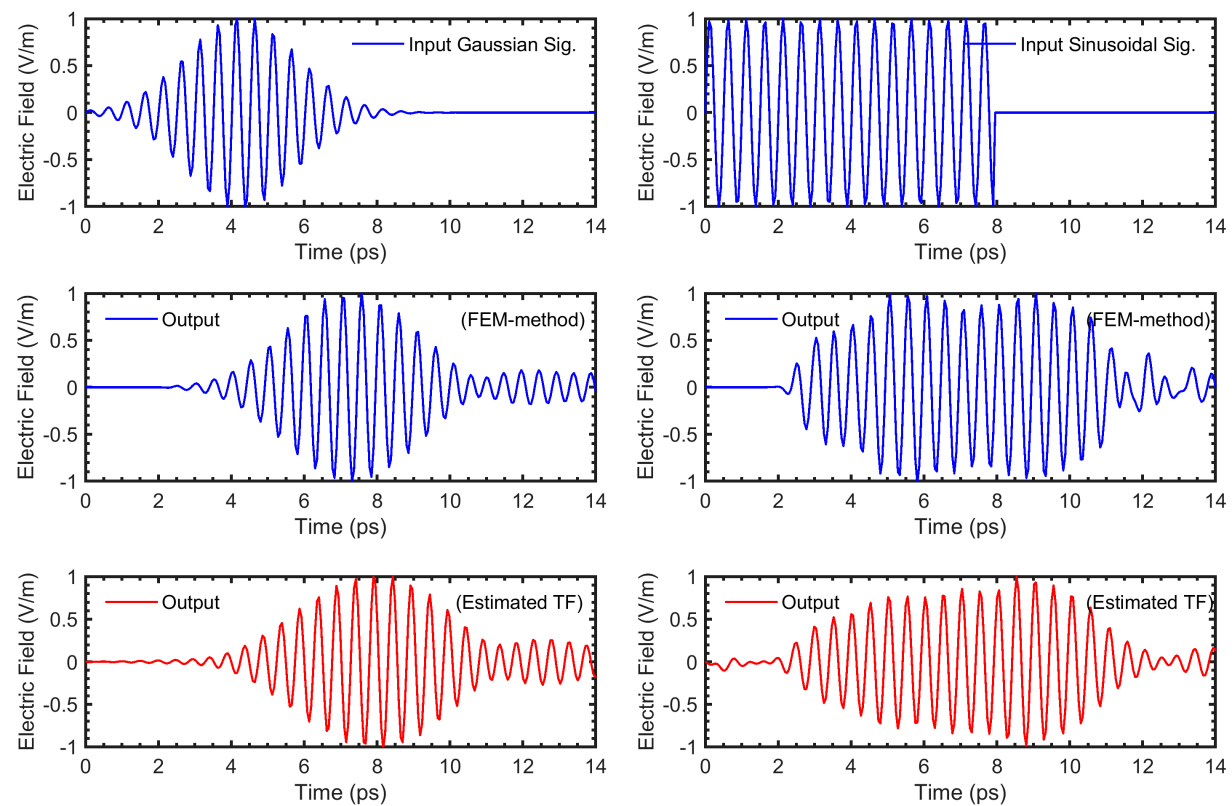

(a)

(b)

Fig. 10 A validation test of the estimated transfer function $\mathrm{H}(\mathrm{s})$ in terms of two different input signals of (a) Gaussian, and (b) sinusoidal pulses, respectively, with the corresponding output waveforms obtained from the FEM analysis of the proposed structure.

known broadband Gaussian pulse which is defined as Equation (9). Figure 9a-b show a time-domain validation test of $\mathrm{H}(\mathrm{s})$ introduced by the coefficients given in Table 1. A good agreement between the full-wave results achieved by the FIT simulation and those of applying the equivalent system function, $\mathrm{H}(\mathrm{s})$, is observed.

$$
E_{y}= \begin{cases}E_{0} e^{-\left(\left(t-0.5 T_{0}\right) / \tau\right)^{2}} \sin \left(2 \pi f_{0} t\right), & \text { if } t \leq T_{0} \\ 0, & \text { otherwise }\end{cases}
$$

where $\tau$ is 2 ps for an appropriate bandwidth compatible with the proposed structure and $T_{0}$ is set to $8 \mathrm{ps}$, the same as previously used in Equation (3). As shown in Figures 10a-b, this approach has great accuracy and could replace the full-wave simulation analysis which is very time consuming. In fact, the TF estimation with the features of the wideband and memory-efficient analysis could be applied in conjunction with other complicated model estimation techniques such as Transmission-Line (TL) models and narrow band equivalent circuit models. 


\section{Conclusions}

This paper provides a standard procedure to examining the performance of a waveguide-based terahertz pulse shaper by introducing the transfer function of the device. The main advantage of this model identification method is that by using a transfer function, the output of the structure can be easily obtained under different input signals in both time and frequency domains. The transfer function was calculated based on the obtained input/output time samples of the full-wave simulations of the proposed $\mathrm{THz}$ waveguide-based pulse compressor. The zeros and poles of the transfer function is determined and a close match between the FEM output and the reconstructed output using the estimated transfer function is obtained.

We confirm that all authors contributed to this manuscript. S.M.R. wrote the initial draft of the manuscript as a part of his $\mathrm{PhD}$ thesis under advisory of R.S and Z.G.H. and the paper has been reviewed prior to submission by the advisors. S.M.R. provided the concept and simulation design of the study. All authors discussed the results, analyzed the data, and commented on the manuscript. All authors have read and agreed to the published version of the manuscript.

Acknowledgements we thankfully acknowledge scientific support and valuable discussions by Miguel Navarro-Cía (University of Birmingham).

\section{Conflict of interest}

The authors declare no conflicts of interest.

\section{References}

1. Dorney, T.D.; Baraniuk, R.G.; Mittleman, D.M., Material parameter estimation with terahertz time-domain spectroscopy, JOSA A, 18, 1562-1571(2001)

2. Peretti, R., Mitryukovskiy, S., Froberger, K., Mebarki, M.A., Eliet, S.,Vanwolleghem, M., and Lampin, J.F., THz-TDS time-trace analysis for the extraction of material and metamaterial parameters, IEEE Trans. Terahertz Sci. Technol., 9, 136-149, (2018)

3. Yi, M.; Kim, H.; Jin, K.H.; Ye, J.C.; Ahn, J., Terahertz substance imaging by waveform shaping, Opt. Express, 20, 20783-20789(2012)

4. Lopato, P.; Chady, T.; Sikora, R.; Gratkowski, S.; Ziolkowski, M., Full wave numerical modelling of terahertz systems for nondestructive evaluation of dielectric structures, COMPEL Int. J. Comput. Math. Electr. Electron. Eng., 32, 736-749(2013)

5. Zhong, H.; Redo-Sanchez, A.; Zhang, X.C., Identification and classification of chemicals using terahertz reflective spectroscopic focal-plane imaging system, Opt. Express, 14, $9130-9141(2006)$

6. Hermelo, M.F.; Shih, P.T.B.; Steeg, M.; Ng'oma, A.; Stöhr, A., Spectral efficient 64QAM-OFDM terahertz communication link, Opt. Express, 25, 19360-19370(2017)

7. Grootendorst, M.R.; Fitzgerald, A.J.; De Koning, S.G.B.; Santaolalla, A.; Portieri, A.; Van Hemelrijck, M.; Young, M.R.; Owen, J.; Cariati, M.; Pepper, M.; et al., Use of a handheld terahertz pulsed imaging device to differentiate benign and malignant breast tissue, Biomed. Opt. Express, 8, 2932-2945(2017) 
8. Gingras, L.; Cooke, D.G., Direct temporal shaping of terahertz light pulses, Optica, 4, $1416-1420(2017)$

9. Keren-Zur, S.; Ellenbogen, T., Direct space to time terahertz pulse shaping with nonlinear metasurfaces, Opt. Express, 27, 20837-20847(2019)

10. Veli, M.; Mengu, D.; Yardimci, N.T.; Luo, Y.; Li, J.; Rivenson, Y.; Jarrahi, M.; Ozcan, A., Terahertz Pulse Shaping Using Diffractive Legos, arXiv, 2006.16599(2020)

11. Razavizadeh, S.M.; Kashani, Z.G.; Sadeghzadeh, R.; Navarro-Cía, M., Tunable compression of $\mathrm{THz}$ chirped pulses using a helical graphene ribbon-loaded hollow-core waveguide, Appl. Opt., 59, 4247-4253(2020)

12. Banos, A.; Gomez, F., Parametric identification of transfer functions from frequency response data, Comput. Control Eng. J., 6, 137-144(1995)

13. Kim, S.; Son, S.U.; Kim, H.; Choi, K.H.; Choi, J.W., Estimate of Passive Time Reversal Communication Performance in Shallow Water, Appl. Sci., 8,23(2018)

14. Zhou, X.; Wang, C.; Tang, R.; Zhang, M., Channel estimation based on statistical frames and confidence level in OFDM systems, Appl. Sci., 8, 1607(2018)

15. Srivastav, P.S.; Chen, L.; Wahla, A.H., Precise Channel Estimation Approach for a mmWave MIMO System, Appl. Sci., 10, 4397(2020)

16. O'Hara, J.F.; Ekin, S.; Choi, W.; Song, I. A perspective on terahertz next-generation wireless communications Technologies, 7, 43, (2019)

17. Taschin, A.; Bartolini, P.; Tasseva, J.; Striova, J.; Fontana, R.; Riminesi, C.; Torre, R., Drawing materials studied by THz spectroscopy, arXiv, 1703.01770(2017)

18. Zaytsev, K.I.; Gavdush, A.A.; Lebedev, S.P.; Yurchenko, S.O., Novel algorithm for sample material parameter determination using $\mathrm{THz}$ time-domain spectrometer signal processing, Journal of Physics: Conference Series, IOP Publishing: Bristol, UK, 486, 012018(2014)

19. Huang, Y.; Sun, P.; Zhang, Z.; Jin, C., Numerical method based on transfer function for eliminating water vapor noise from terahertz spectra, Appl. Opt., 56, 5698 - 5704(2017)

20. Piesiewicz, R.; Jansen, C.; Wietzke, S.; Mittleman, D.; Koch, M.; Kürner, T., Properties of building and plastic materials in the $\mathrm{THz}$ range, Int. J. Infrared Millim. Waves,28, 363$371(2007)$

21. Oral, M.; Long, L.; Bell, R.; Bell, S.; Bell, R.; Alexander, R.; Ward, C., Optical properties of the metals Al., Co Cu Au Fe Pb Ni Pd Pt Ag Ti W Infrared Far Infrared Appl. Opt. , $22,1099-1120(1983)$

22. Macinnes, P.; Ronald, K.; Burt, G.; Cross, A.; Young, A.; Phelps, A.; Konoplev, I.; He, W.; Samsonov, S.; Bratman, V.; et al., RF pulse compression using helically corrugated waveguides , AIP Conference Proceedings, American Institute of Physics: College Park, MD, USA, 807, 474-479 (2006)

23. Bratman, V.; Denisov, G.; Kolganov, N.; Mishakin, S.; Samsonov, S.; Cross, A.; He, W.; Zhang, L.; McStravick, M.; Whyte, C.; et al., Generation of 3 GW microwave pulses in Xband from a combination of a relativistic backward-wave oscillator and a helical-waveguide compressor, Phys. Plasmas, 17, 11703( 2010)

24. Yang, H.Y.; Lin, Y.S.; Chen, C.C., 2.5 dB NF 3.1-10.6 GHz CMOS UWB LNA with small group-delay Variation, Electron. Lett., 44, 528-530(2008)

25. Ullah, S.; Ruan, C.; Sadiq, M.S.; Haq, T.U.; Fahad, A.K.; He, W., Super Wide Band, Defected Ground Structure (DGS), and Stepped Meander Line Antenna for WLAN/ISM/WiMAX/UWB and other Wireless Communication Applications, Sensors, 20, $1735(2020)$

26. Zhang, L.; Mishakin, S.V.; He, W.; Samsonov, S.V.; McStravick, M.; Denisov, G.G.; Cross, A.W.; Bratman, V.L.; Whyte, C.G.; Robertson, C.W.; et al., Experimental study of microwave pulse compression using a five-fold helically corrugated waveguide, IEEE Trans. Microwave Theory Tech., 3, 190-1096(2015)

27. Liu, Y.; Park, S.G.; Weiner, A.,Terahertz waveform synthesis via optical pulse shaping, IEEE J. Sel. Top. Quantum Electron, 2, 79—719(1996) 